\title{
Numerical Comparison between Nonisolated and Isolated Metal-Electrode-Based Dielectrophoresis Cell Separation
}

\author{
Mazdak Rad Malekshahi*, Karim Ansaroudi, \\ Hadi Veladi and Manouchehr Bahrami ${ }^{1}$ \\ Microsystem Fabrication Lab, Faculty of Electrical and Computer Engineering, \\ University of Tabriz, Bolvar 29 Bahman, Tabriz 51666-15813, Iran \\ ${ }^{1}$ Faculty of Electrical and Computer Engineering, University of Tabriz, Bolvar 29 Bahman, \\ Tabriz 51666-15813, Iran
}

(Received June 10, 2013; accepted August 21, 2013)

Key words: dielectrophoresis, cell separation, interdigitated electrode arrays, dielectric layer

The effects of electrode isolation on dielectrophoresis (DEP)-based cell separation devices are discussed. In this study, the conventional dielectrophoresis forces using interdigitated electrode arrays are numerically investigated in the presence of a dielectric layer between electrodes and electrolytes and compared with the direct contact model. It is observed that insertion of a dielectric as an isolation layer affects the spectral response of the DEP forces, as a result of the frequency dependence of the fields. The cells under study are live and dead yeast cells and an isolation layer is developed using polydimethylsiloxane (PDMS) elastomer.

\section{Introduction}

The separation of particles and cells is one of the essential tasks of modern lab-ona-chip (LOC) devices, which are used in diverse research areas including biology and medicine. ${ }^{(1-3)}$ Various active separation methods have been developed in microfluidics, including electrophoresis, ${ }^{(4)}$ magnetic activated cell sorters (MACS) ${ }^{(5)}$ and fluorescent activated cell sorters (FACS). ${ }^{(6)}$ In addition, some passive techniques have also been developed although they have less efficiency than the active ones.

One of the most effective active separation techniques is based on dielectrophoresis (DEP) force, ${ }^{(7)}$ which was firstly introduced by Pohl. ${ }^{(8)}$ DEP is the force applied on natural particles when subject to a nonuniform electric field. Depending on the mutual dielectric properties of the particles and the suspending medium, the DEP force can be either positive or negative. DEP enables the distinct feature of label-free separation, ${ }^{(7)}$ which is based on particles' physical properties such as their size and electrical properties.

*Corresponding author: e-mail: malekshahi@tabrizu.ac.ir 
In most electrode-based DEP devices, various structures of coplanar metal electrodes are devised at the bottom of the microchannel to create a nonuniform electric field, e.g., interdigitated, ${ }^{(9,10)}$ castellated, ${ }^{(11,12)}$ curved $^{(13,14)}$ and polynomial.(15) Metal electrodes are made of precious metals usually requiring multilevel deposition processes; in most cases, electrodes are made from chromium/gold, ${ }^{(16)}$ titanium/gold ${ }^{(17)}$ and titanium/platinum. ${ }^{(18,19)}$ However, the direct contact between metal electrodes and fluid in basic DEP devices would cause some side effects such as electrode corrosion and bubble production, ${ }^{(20,21)}$ and could result in defects during the separation process. On the other hand, thick electrode layers prevent proper bonding between the substrate and the microchannels, and result in channel leakage.

One of the effective solutions to the above issues is to isolate the fluid and the electrodes using a dielectric layer. In ref. 22, a microscope slide was used as an insulation layer over the electrodes, built on a printed circuit board (PCB) and the fluid including HeLa cells and polystyrene particles, and DEP was employed to manipulate them. In ref. 23, electrodes over the wall were separated from the channel by a layer of polydimethylsiloxane (PDMS) elastic polymer; the technique was referred to as contactless DEP and the research conducted to manipulate THP-1 human leukemia monocytes, MCF-7 breast cancer cells, and MCF-10A breast cells using DEP.

In this paper, the main features of isolated-electrode-based DEP separation are investigated numerically and compared with the original nonisolated-electrode-based DEP. For isolated-electrode-based DEP, it is assumed that planar metal electrodes are separated from the medium by coating a PDMS layer. The magnitude and frequency response of the induced DEP force are investigated under the effect of isolation layer thickness. Finally, to assess the device, continuous separation of live and dead yeast cells in both models is carried out under different flow rates.

\section{Theory}

\subsection{Dielectrophoresis}

When a neutral particle is subjected to a nonuniform electric field, it will be polarized by the Maxwell-Wagner interfacial polarization mechanism and DEP force is applied on it. (24) In the case of a homogeneous spherical particle using the effective dipole moment method, ${ }^{(25)}$ the time-averaged DEP force would be as follows. ${ }^{(24)}$

$$
<\boldsymbol{F}_{\mathrm{DEP}}>=\pi \varepsilon_{\mathrm{m}} r^{3} \operatorname{Re}[K(\omega)] \nabla|\boldsymbol{E}|^{2}
$$

$\boldsymbol{E}$ is the applied electric field, and as shown by the expression, the force depends on the gradient of the field magnitude instead of its variation. Thus, a nonuniform field is required to induce the DEP forces. $K(\omega)$ is the Clausius-Mossotti (CM) factor and defined as

$$
K(\omega)=\frac{\tilde{\varepsilon}_{\mathrm{p}}-\tilde{\varepsilon}_{\mathrm{m}}}{\tilde{\varepsilon}_{\mathrm{p}}+2 \tilde{\varepsilon}_{\mathrm{m}}},
$$

where $\tilde{\varepsilon}_{\mathrm{p}}$ and $\tilde{\varepsilon}_{\mathrm{m}}$ denote complex permittivities of particle and fluid and obtained as 


$$
\tilde{\varepsilon}=\varepsilon-j \frac{\sigma}{\omega},
$$

where $\varepsilon$ and $\sigma$ are permittivity and conductivity, respectively; $\omega$ shows field angular frequency.

As eq. (1) shows, the applied field attracts the particles to the locations with the highest electric field gradient when $\operatorname{Re}[K(\omega)]>0$, known as positive dielectrophoresis (pDEP). On the other hand, when $\operatorname{Re}[K(\omega)]<0$, the fluid polarization is more dominant, which results in particle repulsion from the same points, and is called negative dielectrophoresis (nDEP). This effect can be used in cell separators to align various particles based on their electrical properties. In most cases, the complex permittivity of the cells can be estimated using a multi-shell model.(25)

According to eq. (1), crossover frequency is a frequency in which the real value of the $\mathrm{CM}$ factor is zero, which is defined as

$$
f_{\text {crossover }}=\frac{1}{2 \pi} \sqrt{\frac{\left(\sigma_{\mathrm{m}}-\sigma_{\mathrm{p}}\right)\left(\sigma_{\mathrm{p}}+2 \sigma_{\mathrm{m}}\right)}{\left(\varepsilon_{\mathrm{p}}-\varepsilon_{\mathrm{m}}\right)\left(\varepsilon_{\mathrm{p}}+2 \varepsilon_{\mathrm{m}}\right)}} .
$$

\subsection{Fluid dynamic}

For an incompressible fluid flow inside a microchannel, its profile is given by the Navier-Stokes equation in the low Reynolds number approximation and can be expressed as $^{(26)}$

$$
\begin{gathered}
\nabla \cdot \boldsymbol{u}=0, \\
-\nabla p+\eta \nabla^{2} \boldsymbol{u}=0,
\end{gathered}
$$

where $p$ is pressure, $\eta$ is medium viscosity, and $\boldsymbol{u}$ is medium velocity. The hydrodynamic drag force $\boldsymbol{F}_{\text {drag }}$ that acts on the spherical particles of radius $\mathrm{r}$ is calculated from the Stokes law, which is given by ${ }^{(27)}$

$$
\boldsymbol{F}_{\text {drag }}=6 \pi \eta r\left(\boldsymbol{v}_{\text {particle }}-\boldsymbol{u}\right),
$$

where $\boldsymbol{v}_{\text {particle }}$ is the particle velocity and $\boldsymbol{u}$ is the medium velocity.

\section{Materials and Methods}

\subsection{Design of models}

To compare the nonisolated- and isolated-electrode-based DEPs, two alternative models have been introduced as shown in Fig. 1.

Both models used an interdigitated electrode array to generate the required nonuniform field along the channel. The electrodes are $100 \mu \mathrm{m}$ in width and separated by $80 \mu \mathrm{m}$. The required microchannels are developed in PDMS, which is directly bonded to the substrate in a nonisolated model. For the isolated model on the other hand, the PDMS is also applied at the bottom of the channel to provide the required isolation from 


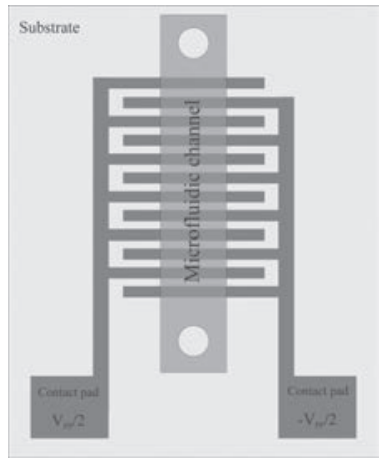

(a)

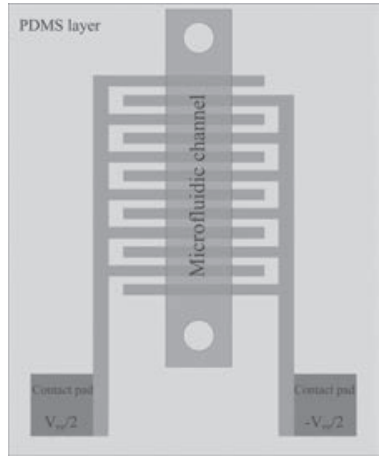

(b)

Fig. 1. Schematic of interdigitated electrode and microchannel for (a) nonisolated and (b) isolated DEP separation models.

electrodes and adjusted to the target thickness $\left(T_{\mathrm{PDMS}}\right)$ by the spinning speed. PDMS has high chemical stability, excellent biocompatibility, and is optically transparent, making it a desirable biochip material. ${ }^{(28,29)}$ Both models are assumed to have a similar channel height $\left(H_{c}\right)$ of $40 \mu \mathrm{m}$ and width of $200 \mu \mathrm{m}$.

\subsection{Property of samples}

Using the proposed model, the dielectrophoresis-based separation technique has been investigated using live and dead yeast cells. To determine the CM factors for the mentioned cells, they are modelled using two-layered spherical shells. ${ }^{(25)}$ The required parameters of both cell models were obtained from ref. 30 .

The frequency spectrum of $\operatorname{Re}[K(\omega)]$ for live and dead yeast cells suspended in a buffer solution of $2.8 \mathrm{mS} / \mathrm{m}$ electrical conductivity and relative permittivity of 78 is shown in Fig. 2. It is found that the live cells experience two crossover frequencies where the real part of the CM factor is zero. The lower crossover is $18.78 \mathrm{kHz}$ and the higher one is $44.56 \mathrm{MHz}$; the $\mathrm{CM}$ factor between the two crossovers is positive; otherwise, it is negative. The dead yeast cells, on the other hand, show a single crossover at $1.772 \mathrm{MHz}$. This suggests a significant dissimilarity in the behaviour of viable and nonviable cells during a DEP process and can be effectively used in cell separation.

\subsection{Simulation requirements}

For the models described above, the electric and fluid fields were calculated using the finite element method software (COMSOL Multiphysics ${ }^{\mathrm{TM}}$ ). The subdomain settings and constants used for the simulation are presented in Table 1.

Since the electrodes are periodically located as shown in Fig. 1, only a single pair of electrodes is considered for Finite Element Analysis (FEA) simulation. As the electrode is sufficiently long with respect to its width and separations, we only exploited a $2 \mathrm{D}$ model to reduce the model complexity. Also, the thickness of electrodes is not considered $\left(T_{\mathrm{E}}\right.$ $=0)$. 


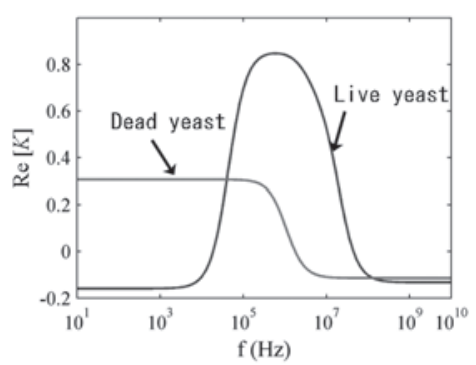

Fig. 2. Frequency spectra of $\operatorname{Re}[K]$ for live and dead yeast cells.

Table 1

Properties used in the simulations.

\begin{tabular}{lc}
\hline Property & Value \\
\hline Dynamic viscosity of fluid & $1 \times 10^{-3} \mathrm{~Pa} \cdot \mathrm{s}$ \\
Density of fluid & $1000 \mathrm{~kg} / \mathrm{m}^{3}$ \\
Relative permittivity of fluid & $1000 \mathrm{~kg} / \mathrm{m}^{3}$ \\
Conductivity of fluid & $28 \times 10^{-3} \mathrm{~S} / \mathrm{m}$ \\
Relative permittivity of PDMS & 2.65 \\
Conductivity of PDMS & $0.83 \times 10^{-12} \mathrm{~S} / \mathrm{m}$ \\
Permittivity of free space $\left(\varepsilon_{0}\right)$ & $8.854 \times 10^{-12} \mathrm{~F} / \mathrm{m}$ \\
Electrode peak-to-peak voltage $\left(V_{\mathrm{PP}}\right)$ & $40 \mathrm{~V}$ \\
Frequency & Variable \\
\hline
\end{tabular}

Initially, the potential is calculated along the channel using the Laplace equation. Owing to the low density of particles involved, their effects on potential distribution are negligible. In addition, the interactions between particles are not considered. The potential gradient $(\partial \varphi / \partial n)$ is set to zero owing to the channel's dielectric property and also from the continuity of the normal current density over the interface of PDMS and fluid.

The dynamic fluid velocity along the channel was determined by solving the NavierStokes equation together with continuity equations. The necessary boundary conditions for the Navier-Stokes equation include known velocity at the inlet and also zero pressure at the outlet of the microchannel. In addition, the normal components of velocity are zero (no slip) over the walls and the electrodes. The required boundary conditions for two models are described in Fig. 3.

\subsection{Simulation of particle tracing}

The dynamic behaviour of the dedicated cells can then be determined using Newton's second law of thermodynamics. The forces over the cells include DEP, hydrodynamic drag force $\boldsymbol{F}_{\text {drag }}$ and sedimentation force. Sedimentation force is negligible as the density of particles is close to that of the suspending buffer solution. 


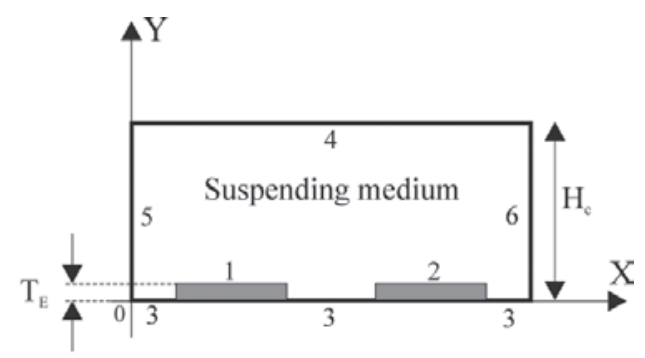

\begin{tabular}{ccc}
\hline & \multicolumn{2}{c}{ Boundary conditions } \\
\cline { 2 - 3 } Boundary & Electric Field & Flow Field \\
\hline 1 & $V_{\mathrm{pp}} / 2$ & No slip \\
2 & $-V_{\mathrm{pp}} / 2$ & No slip \\
3 & Electric insulation & No slip \\
4 & Electric insulation & No slip \\
5 & Periodic & Velocity \\
6 & Periodic & $P_{0}=0$ \\
\hline
\end{tabular}

(a)

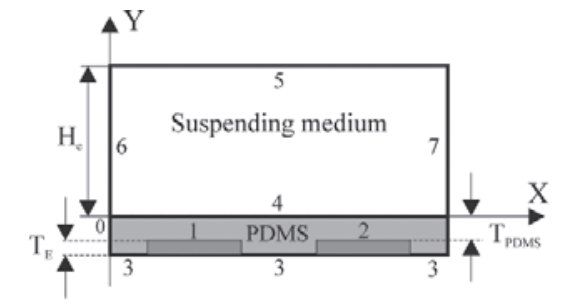

\begin{tabular}{ccc}
\hline \multirow{2}{*}{ Boundary } & \multicolumn{2}{c}{ Boundary conditions } \\
\cline { 2 - 3 } & Electric Field & Flow Field \\
\hline 1 & $V_{\mathrm{pp}} / 2$ & $-*$ \\
2 & $-V_{\mathrm{pp}} / 2$ & - \\
3 & Electric insulation & - \\
4 & Continuity & No slip \\
5 & Electric insulation & No slip \\
6 & Periodic & Velocity \\
7 & Periodic & $P_{0}=0$ \\
\hline *Not active in this domain &
\end{tabular}

(b)

Fig. 3. Boundary conditions for (a) nonisolated and (b) isolated DEP separation models.

$$
m \frac{\mathrm{d}^{2} r}{\mathrm{~d} t^{2}}=\boldsymbol{F}_{\mathrm{DEP}}+\boldsymbol{F}_{\mathrm{drag}}
$$

Owing to the small acceleration time, the inertia of the cells can be neglected. Therefore, the particle velocity is determined by the equilibrium of the DEP force and the drag force.

$$
\boldsymbol{v}_{\text {particle }}=\frac{\boldsymbol{F}_{\mathrm{DEP}}}{6 \pi \eta r}+\boldsymbol{u}
$$

Using postprocessing tools and exporting the results to MATLAB ${ }^{\mathrm{TM}}$, simulated particles can be traced in the flow with specified flow rates, and the trapping efficiency of both models can be compared.

\section{Results and Discussion}

\subsection{Frequency spectrum of gradient in microchannel}

The spectra of the squared field gradient $\left(\nabla E^{2}\right)$ in a dedicated point $\mathrm{A}(140 \mu \mathrm{m}, 10$ $\mu \mathrm{m})$ for both models in the frequency range of $10^{1}-10^{10} \mathrm{~Hz}$ are plotted in Figs. 4(a) and 4(b).

As the plots show, the gradient in the nonisolated model shows a flat response in the specific range as the medium represents a constant resistive behaviour with no significant 


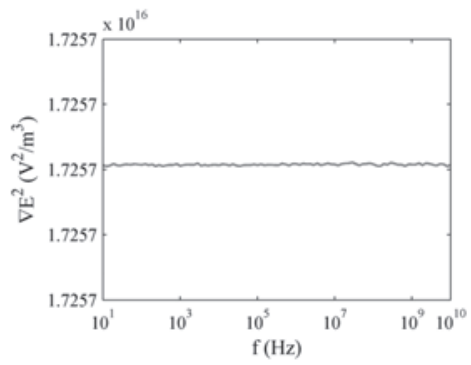

(a)

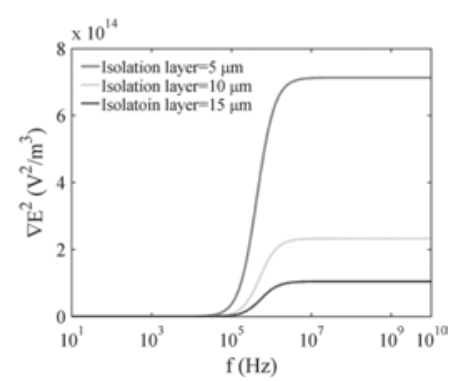

(b)

Fig. 4. Frequency spectra of squared field gradient for (a) nonisolated and (b) isolated models.

frequency dependence.

For the isolated model, on the other hand, a negligible field is induced at low frequencies, which gradually increased with increasing frequency, suggesting a high pass filter behaviour for the structure. This arises from the capacitive function created by a dielectric layer embedded between electrodes and the electrolyte.

The results revealed that a thin layer results in a large capacitance and, therefore, the amplitudes are also greater at the pass band. However, the cutoff frequencies are approximately constant for different thicknesses. The latter firmly depends on the fluid electrical conductivity, which is assumed to be constant throughout this study. Thus a relatively constant cutoff frequency is achieved for all models.

\subsection{Spatial distribution of gradient in microchannel}

Using the above discussion, the surface plots of gradients for both isolated and nonisolated models were obtained at $f=10 \mathrm{MHz}$ and the results are shown in Fig. 5. In both models, the maximum gradient along the y axes appears around the electrode edges, and this extreme amount is decreasing with increased isolation.

\subsection{DEP force in two models}

Eventually, it is possible to compare the dielectrophoresis forces in both devices. Figure 6 shows DEP force imposed on the yeast cells for both nonisolated and isolated devices at given point A. By comparing Fig. 6(a) with Figs. 6(b)-6(d), it is concluded that, in the nonisolated model, only the CM factor is the dominant factor over the frequency response; for the isolated device, the frequency response depends on both the field gradient and CM factor. In the latter case, the frequency response vanishes at low frequencies owing to the capacitive effects of the isolation layer. We referred to this as zero DEP (zDEP) in the rest of this paper. The response is almost similar in all models at larger frequencies besides the reduced amplitude as the insertion loss of dielectric layers. One could also observe the main difference in the isolated model, where at low frequencies, both live and dead cells experience zDEP.

For a more convenient comparison between the discussed models, the ratios of DEP force in isolated to nonisolated models of different thicknesses are shown in Fig. 7. 


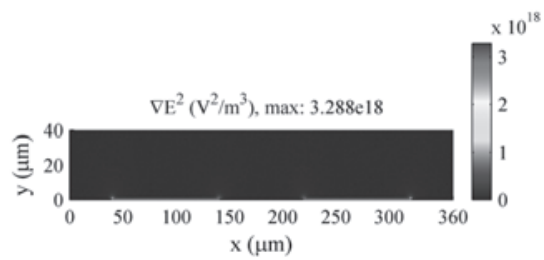

(a)

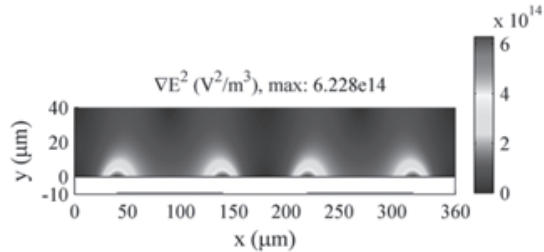

(c)

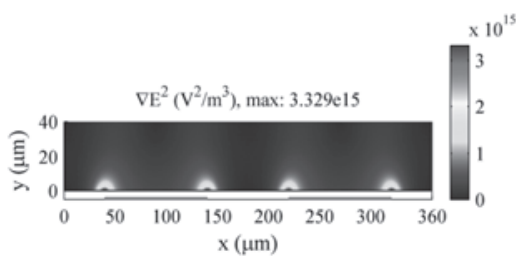

(b)

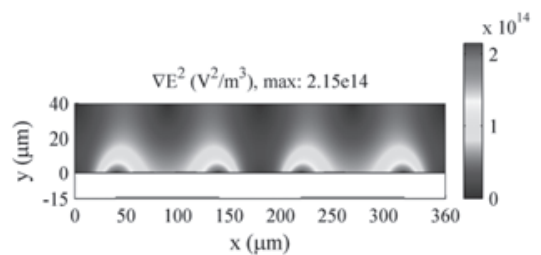

(d)

Fig. 5. Surface plot in microchannel for (a) nonisolated and isolated electrodes with (b) $5 \mu \mathrm{m}$ isolating layer thickness, (c) $10 \mu \mathrm{m}$ isolating layer thickness, and (d) $15 \mu \mathrm{m}$ isolating layer thickness (White areas stand for out-of-scale values).

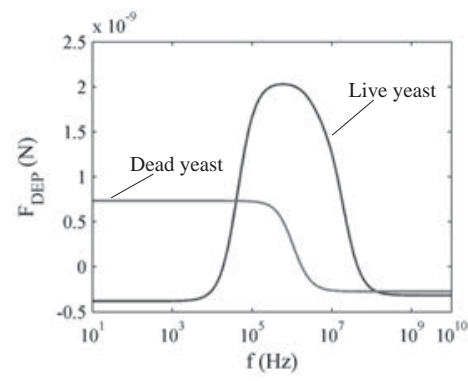

(a)

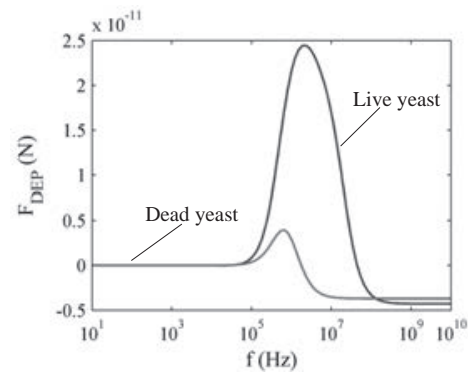

(c)

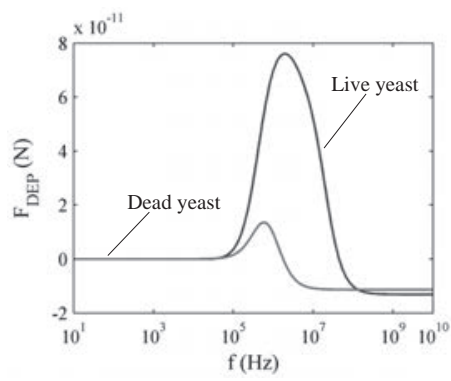

(b)

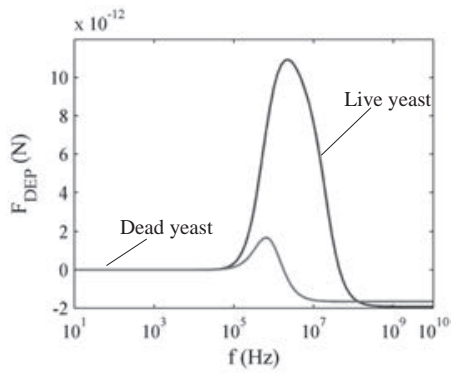

(d)

Fig. 6. Frequency response of DEP force imposed on yeast cells at the dedicated point A for (a) nonisolated-electrode-based DEP and isolated-electrode-based DEP with (b) $5 \mu \mathrm{m}$ isolating layer thickness, (c) $10 \mu \mathrm{m}$ isolating layer thickness, and (d) $15 \mu \mathrm{m}$ isolating layer thickness. 


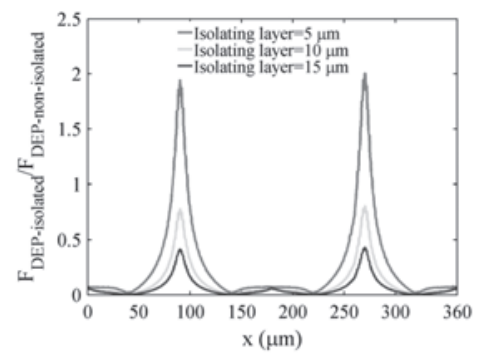

(a)

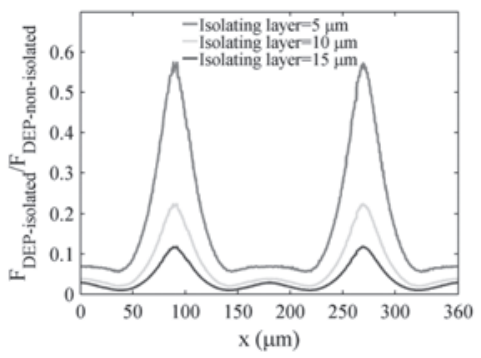

(c)

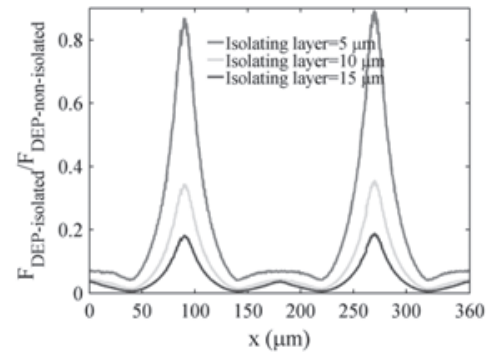

(b)

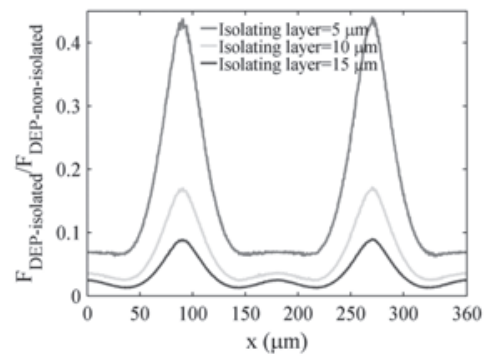

(d)

Fig. 7. Ratio of $F_{\text {DEP-isolated }}$ to $F_{\text {DEP-nonisolated }}$ at $f=10 \mathrm{MHz}$ in (a) height $=5 \mu \mathrm{m}$, (b) height $=10 \mu \mathrm{m}$, (c) height $=15 \mu \mathrm{m}$, and (d) height $=20 \mu \mathrm{m}$ on live yeast cells.

\subsection{Optimum frequency for separation of cells}

For the separation of live and dead yeast cells, the flow separation (DEP affinity) strategy is used.(20) A suspension containing a mixture of live and dead cells is injected into the microfluidic channel. The main goal is to trap the desired cells at the bottom of the microfluidic channel while the others are repelled and can then be washed off. To achieve this, the cells should receive different types of DEP, i.e. nDEP and pDEP, which can be achieved by selecting appropriate stimulation frequencies.

The frequency range of the DEP force for nonisolated electrodes is summarized from Fig. 6(a) in Table 2. As highlighted in the table, two distinct frequency ranges exist where the live and dead cells experience different DEP forces. For the dedicated cell combination and fluid characteristics, at frequencies from DC to $18.78 \mathrm{kHz}$, dead cells receive pDEP, which causes the cells to be trapped around the electrodes while the live cells are repelled by nDEP. The situation is reversed at the frequency window of 1.7744.56 MHz. In all other frequencies, both cells are subjected to the same type of DEP force. The frequency range of the DEP force for isolated electrodes is also shown in Table 3, where a single window is obtained in $1.77-44.56 \mathrm{MHz}$, which can be employed to selectively manipulate the different cell types. To be able to compare the models, a second frequency window is considered for cell separation in the following sections. 
Table 2

Frequency range of DEP force for nonisolated electrodes.

\begin{tabular}{lcc}
\hline \multirow{2}{*}{ Frequency range } & \multicolumn{2}{c}{ Nonisolated-electrode-based DEP } \\
\cline { 2 - 3 } & Live yeast & Dead yeast \\
\hline$<\mathbf{1 8 . 7 8} \mathbf{~ k H z}$ & Negative DEP & Positive DEP \\
$\mathbf{1 8 . 7 8}-1.77 \mathrm{MHz}$ & Positive DEP & Positive DEP \\
$\mathbf{1 . 7 7 - 4 4 . 5 6 ~} \mathbf{~ M H z}$ & Positive DEP & Negative DEP \\
$>44.56 \mathrm{MHz}$ & Negative DEP & Negative DEP \\
\hline
\end{tabular}

Table 3

Frequency range of DEP force for isolated electrodes.

\begin{tabular}{lcc}
\hline \multirow{2}{*}{ Frequency range } & \multicolumn{2}{c}{ Isolated-electrode-based DEP } \\
\cline { 2 - 3 } & Live yeast & Dead yeast \\
\hline$<49.5 \mathrm{kHz}$ & Zero DEP & Zero DEP \\
$49.5-1.77 \mathrm{MHz}$ & Positive DEP & Positive DEP \\
$\mathbf{1 . 7 7 - 4 4 . 5 6 ~ M H z}$ & Positive DEP & Negative DEP \\
$>44.56 \mathrm{MHz}$ & Negative DEP & Negative DEP \\
\hline
\end{tabular}

\subsection{Effect of flow rate on cell separation}

Sample throughput is one of the main factors in the proper operation of a microfluidic device in cell separation. The flow rate directly depends on fluid flow rates. Generally, the higher the flow rate, the larger the throughput, and thus the separation process can be faster. However, to cope with a higher transport velocity of samples, the required DEP forces should be proportionally increased so that the hydrodynamic drag force and DEP can be matched properly. Naturally, this would limit the flow rate.

In practise, flow rates can be optimised to achieve the best performance. At the lower flow rates, dead yeast cells could not completely pass through the channel while at higher flow rates, they would not be properly trapped in the channel.

Figure 8 shows the predicted trajectories of live and dead yeast cells in the nonisolated device in different flow rates where extreme values would be obtained as $Q_{\min }=2.4 \mu \mathrm{l} / \mathrm{min}$ and $Q_{\max }=7.5 \mu \mathrm{l} / \mathrm{min}$. $Q_{\min }$ is mainly limited by repelling cells. If the flow rate is lower than $Q_{\min }$, the unwanted cells cannot exit the channel. On the other hand, $Q_{\max }$ is limited by the trapping cells where very high rates disturb the cell trapping, and therefore, the separation efficiency is reduced.

In the isolated model with a $5 \mu \mathrm{m}$ isolating layer, the cell trajectories for different flow rates are shown in Fig. 9. Using a similar discussion, one could achieve $Q_{\min }=0.3$ $\mu \mathrm{l} / \mathrm{min}$ and $Q_{\max }=0.8 \mu \mathrm{l} / \mathrm{min}$.

In comparison, the throughput of the nonisolating model is higher than that of the isolating model. In addition, increasing the thickness of the PDMS layer results in a lower throughput.

\subsection{Trapping efficiency of yeast cells}

By counting the number of trapped and escaped live yeast cells, the efficiency of arrested live yeast cells can be calculated as 


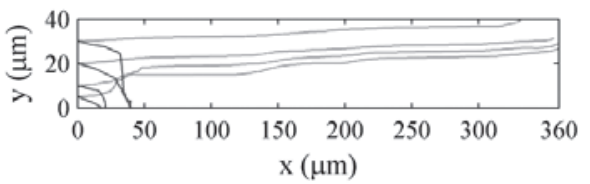

(a)

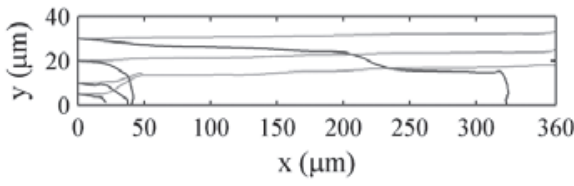

(c)

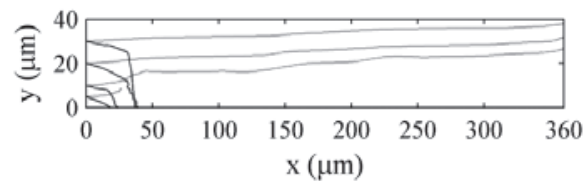

(b)

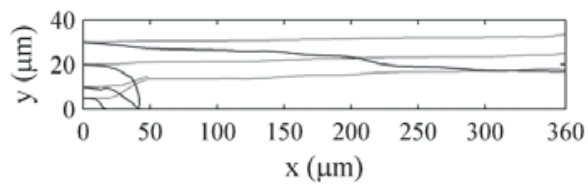

(d)

Fig. 8. Live (dark gray) and dead yeast (light gray) cell trajectrories for nonisolated electrodes at $f=9.8 \mathrm{MHz}$ for (a) $Q=2.3 \mu \mathrm{l} / \mathrm{min}$, (b) $Q=2.4 \mu \mathrm{l} / \mathrm{min}$, (c) $Q=7.5 \mu \mathrm{l} / \mathrm{min}$, and (d) $Q=7.6 \mu \mathrm{l} / \mathrm{min}$.

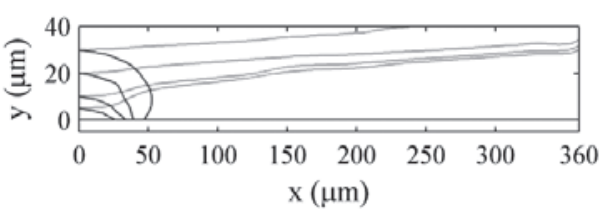

(a)

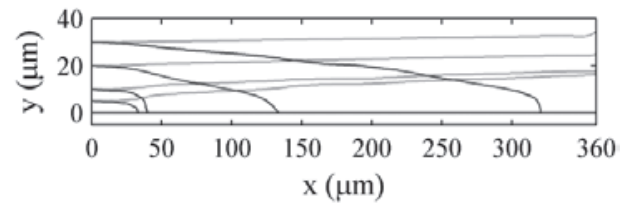

(c)

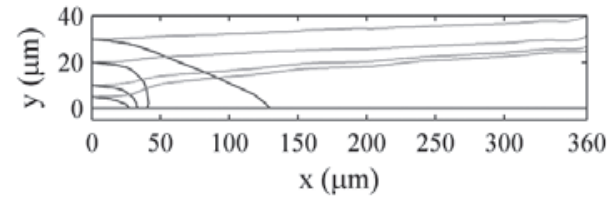

(b)

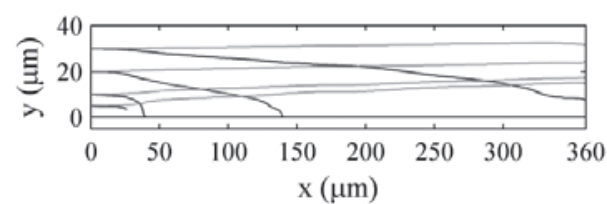

(d)

Fig. 9. Live (dark gray) and dead yeast (light gray) cell trajectrories for isolated electrodes at $f=9.8$ MHz in $5 \mu \mathrm{m}$ isolating layer: (a) $Q=0.2 \mu \mathrm{l} / \mathrm{min}$, (b) $Q=0.3 \mu \mathrm{l} / \mathrm{min}$, (c) $Q=0.8 \mu \mathrm{l} / \mathrm{min}$, and (d) $Q=0.9$ $\mu \mathrm{l} / \mathrm{min}$.

$$
\text { Trapping efficiency }(\%)=\frac{\text { Trapped live cells }}{\text { Trapped live cells }+ \text { Escaped live cells }} \times 100 \%
$$

assuming that 40 yeast cells are uniformly placed at the inlet and the particles are traced. The resulting trapping efficiency is illustrated for different combinations in Fig. 10. For the nonisolated model, the efficiency is flat with increased flow rates up to 5 $\mu \mathrm{l} / \mathrm{min}$, while a much smaller turning point appears for the isolated device, which also becomes smaller for thicker isolation layers.

The above deficiency can be partly improved by involving more electrodes during the simulation. Figure 11 shows the case when two or three pairs of electrodes are considered. As shown in the figure, a more efficient separation process would be achieved by multiple pairs of electrode-isolated devices. 


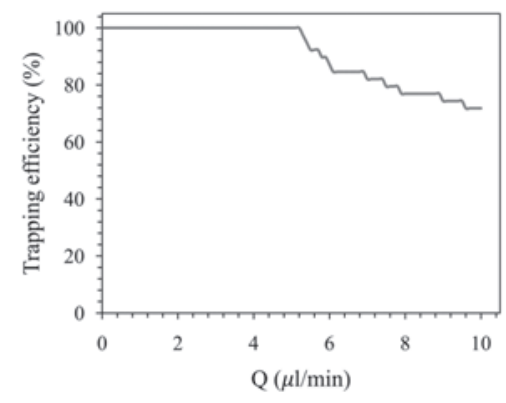

(a)

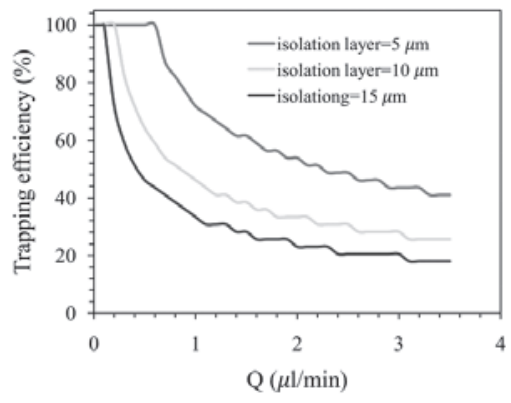

(b)

Fig. 10. Trapping efficiency at $f=9.8 \mathrm{MHz}$ for (a) nonisolated and (b) isolated models with different isolating layers.

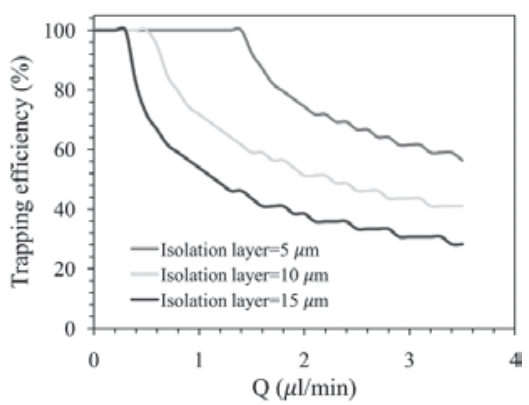

(a)

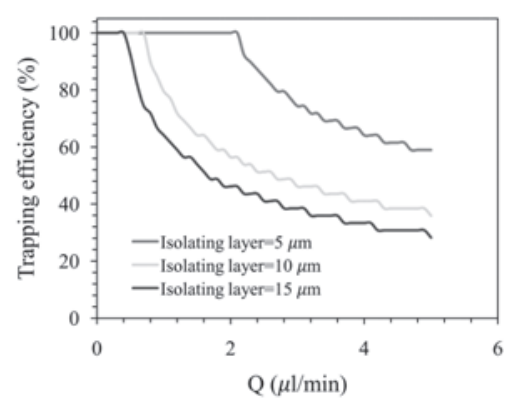

(b)

Fig. 11. Trapping efficiency at $f=9.8 \mathrm{MHz}$ of isolated model for (a) two and (b) three pairs of electrodes.

\section{Conclusions}

The effects of electrode isolation on DEP devices are discussed in detail. By using two models, one with no dielectric layer and the other with an integrated PDMS isolation layer, the generated DEP forces and their frequency response are obtained and compared. Generally, in the absence of isolation, the electrodes are exposed to corrosion during the time. However, we obtained a more configuration-based response in the isolated model together with no corrosion-based device degradation. Another dominant factor is the stimulation frequency, for which one may choose proper values where alternative DEP forces could be used for different cells. The flow rate is an essential factor affecting the separation efficiency and we obtained extreme values for this using a particle-tracing tool. Generally, it is proposed that by using inexpensive metal electrodes such as copper and applying a biocompatible insulating layer such as PDMS on the electrodes, it is possible to fabricate cost-effective DEP-based microparticle separators. 


\section{References}

1 A. A. S. Bhagat, H. Bow, H. W. Hou, S. J. Tan, J. Han and C. T. Lim: Med. Biol. Eng. Comput. 48 (2010) 999.

2 N. Pamme: Lab Chip 7 (2007) 1644.

3 A. Lenshof and T. Laurell: Chem. Soc. Rev. 39 (2010) 1203.

4 A. Marziali, J. Pel, D. Bizzotto and L. A. Whitehead: Electrophoresis 26 (2005) 82.

5 I. Safarık and M. Safarıkova: J. Chromatogr. B 722 (1999) 33.

6 L. A. Herzenberg, D. Parks, B. Sahaf, O. Perez, M. Roederer and L. A. Herzenberg: Clin. Chem. 48 (2002) 1819.

7 P. R. Gascoyne and J. Vykoukal: Electrophoresis 23 (2002) 1973.

8 H. A. Pohl and H. Pohl: Dielectrophoresis: the Behavior of Neutral Matter in Nonuniform Electric Fields (Cambridge University Press, Cambridge, 1978).

9 H. Li and R. Bashir: Biomed. Microdevices 6 (2004) 289.

10 N. Crews, J. Darabi, P. Voglewede, F. Guo and A. Bayoumi: Sens. Actuators, B 125 (2007) 672.

11 F. F. Becker, X.-B. Wang, Y. Huang, R. Pethig, J. Vykoukal and P. Gascoyne: PNAS 92 (1995) 860.

12 W. Mike Arnold and N. R. Franich: Curr. Appl. Phys. 6 (2006) 371.

13 K. Khoshmanesh, C. Zhang, F. J. Tovar-Lopez, S. Nahavandi, S. Baratchi, K. Kalantar-Zadeh and A. Mitchell: Electrophoresis 30 (2009) 3707.

14 K. Khoshmanesh, C. Zhang, F. J. Tovar-Lopez, S. Nahavandi, S. Baratchi, A. Mitchell and K. Kalantar-Zadeh: Microfluid. Nanofluid. 9 (2010) 411.

15 F. Grom, J. Kentsch, T. Müller, T. Schnelle and M. Stelzle: Electrophoresis 27 (2006) 1386.

16 C. L. Asbury, A. H. Diercks and G. van den Engh: Electrophoresis 23 (2002) 2658.

17 H. Morgan, M. P. Hughes and N. G. Green: Biophys. J. 77 (1999) 516.

18 A. R. Minerick, R. Zhou, P. Takhistov and H. C. Chang: Electrophoresis 24 (2003) 3703.

19 H. Li, Y. Zheng, D. Akin and R. Bashir: Microelectromech. Syst. 14 (2005) 103.

20 M. P. Hughes: Electrophoresis 23 (2002) 2569.

21 S. Hardt and F. Schönfeld: Microfluidic Technologies for Miniaturized Analysis Systems (Springer, Boston, 2007).

22 K. Park, H.-J. Suk, D. Akin and R. Bashir: Lab Chip 9 (2009) 2224.

23 H. Shafiee, J. L. Caldwell, M. B. Sano and R. V. Davalos: Biomed. Microdevices 11 (2009) 997.

24 H. Morgan and N. G. Green: AC Electrokinetics: Colloids and Nanoparticles (Research Studies Press, Philadelphia, 2003).

25 T. B. Jones: Electromechanics of Particles (Cambridge University Press, New York, 2005).

26 D. Li: Electrokinetics in Microfluidics (Academic Press, London, 2004).

27 A. Goldman, R. Cox and H. Brenner: Chem. Eng. Sci. 22 (1967) 653.

28 J. Brugger, G. Beljakovic, M. Despont, H. Biebuyck, N. De Rooij and P. Vettiger: Sens. Actuators, A 70 (1998) 191.

29 A. Mata, A. J. Fleischman and S. Roy: Biomed. Microdevices 7 (2005) 281.

30 Y. Huang, R. Holzel, R. Pethig and X.-B. Wang: Phys. Med. Biol. 37 (1992) 1499. 